\title{
On the hierarchical variational inclusion problems in Hilbert spaces
}

\author{
Shih-sen Chang ${ }^{1}$, Jong Kyu Kim² ${ }^{2}$, HW Joseph Lee ${ }^{3}$ and Chi Kin Chun ${ }^{3}$
}

"Correspondence:

jongkyuk@kyungnam.ac.kr

${ }^{2}$ Department of Mathematics

Education, Kyungnam University,

Changwon, Gyeongnam 631-701 Korea

Full list of author information is

available at the end of the article

\begin{abstract}
The purpose of this paper is by using Maingé's approach to study the existence and approximation problem of solutions for a class of hierarchical variational inclusion problems in the setting of Hilbert spaces. As applications, we solve the convex programming problems and quadratic minimization problems by using the main theorems. Our results extend and improve the corresponding recent results announced by many authors.
\end{abstract}

MSC: 47J05; 47H09; 49J25

Keywords: hierarchical variational inclusion problem; hierarchical variational inequality problem; hierarchical optimization problems; quasi-nonexpansive mapping; strongly quasi-nonexpansive mapping; demiclosed principle

\section{Introduction}

Throughout this paper, we assume that $H$ is a real Hilbert space, $C$ is a nonempty closed and convex subset of $H$ and denote by $\operatorname{Fix}(T)$ the set of fixed points of a mapping $T: C \rightarrow C$.

Let $A: H \rightarrow H$ be a single-valued nonlinear mapping and let $M: H \rightarrow 2^{H}$ be a multivalued mapping. The so-called quasi-variational inclusion problem (see [1-3]) is to find a point $u \in H$ such that

$$
\theta \in A(u)+M(u)
$$

A number of problems arising in structural analysis, mechanics and economics can be considered in the framework of this kind of variational inclusions (see, for example, [4]).

The set of solutions of the variational inclusion (1.1) is denoted by $\Omega$.

\section{Special cases}

(I) If $M=\partial \phi: H \rightarrow 2^{H}$, where $\phi: H \rightarrow \mathcal{R} \cup\{+\infty\}$ is a proper convex and lower semicontinuous function and $\partial \phi$ is the sub-differential of $\phi$, then variational inclusion problem

(1.1) is equivalent to finding $u \in H$ such that

$$
\langle A(u), v-u\rangle+\phi(y)-\phi(u) \geq 0, \quad \forall y \in H,
$$

which is called the mixed quasi-variational inequality.

\section{Springer}

(c) 2013 Chang et al:; licensee Springer. This is an Open Access article distributed under the terms of the Creative Commons Attribution License (http://creativecommons.org/licenses/by/2.0), which permits unrestricted use, distribution, and reproduction in any medium, provided the original work is properly cited. 
Especially, if $A=0$, then (1.2) is equivalent to the minimizing problem of $\phi$ on $H$, i.e., to find $u \in H$ such that $\phi(u)=\inf _{y \in H} \phi(y)$.

(II) If $M=\partial \delta_{C}$, where $C$ is a nonempty closed and convex subset of $H$ and $\delta_{C}: H \rightarrow$ $[0, \infty]$ is the indicator function of $C$, i.e.

$$
\delta_{C}(x)= \begin{cases}0, & x \in C, \\ +\infty, & x \notin C,\end{cases}
$$

then variational inclusion problem (1.2) is equivalent to finding $u \in C$ such that

$$
\langle A(u), v-u\rangle \geq 0, \quad \forall v \in C .
$$

This problem is called Hartman-Stampacchia variational inequality problem.

(III) If $M=0$ and $A=I-T$ where $I$ is an identity mapping and $T: H \rightarrow H$ is a nonlinear mapping, then problem (1.1) is equivalent to the fixed point problem of $T$. That is, find $u \in H$ such that

$$
u=T u \text {. }
$$

Recently, hierarchical fixed point problems, hierarchical optimization problems and hierarchical minimization problems have attracted many authors' attention due to their link with convex programming problems, optimization problems and monotone variational inequality problems etc. (see [5-21] and others).

The purpose of this paper is to introduce and study the following bi-level hierarchical variational inclusion problem in the setting of Hilbert spaces:

Find $\left(x^{*}, y^{*}\right) \in \Omega_{1} \times \Omega_{2}$ such that for given positive real numbers $\rho$ and $\eta$, the following inequalities hold:

$$
\begin{cases}\left\langle\rho F\left(y^{*}\right)+x^{*}-y^{*}, x-x^{*}\right\rangle \geq 0, & \forall x \in \Omega_{1}, \\ \left\langle\eta F\left(x^{*}\right)+y^{*}-x^{*}, y-y^{*}\right\rangle \geq 0, & \forall y \in \Omega_{2},\end{cases}
$$

where $F, A_{1}, A_{2}: H \rightarrow H$ are mappings and $M_{1}, M_{2}: H \rightarrow 2^{H}$ are multi-valued mappings, $\Omega_{i}$ is the set of solutions to variational inclusion problem (1.1) with $A=A_{i}, M=M_{i}$ for $i=1,2$.

\section{Special examples}

(I) If $M_{i}=0, A_{i}=I-T_{i}$, where $T_{i}: H \rightarrow H$ is a nonlinear mapping for each $i=1,2$, then $\Omega_{i}=\operatorname{Fix}\left(T_{i}\right)$ and bi-level hierarchical variational inclusion problem (1.5) is equivalent to finding $\left(x^{*}, y^{*}\right) \in \operatorname{Fix}\left(T_{1}\right) \times \operatorname{Fix}\left(T_{2}\right)$ such that

$$
\begin{cases}\left\langle\rho F\left(y^{*}\right)+x^{*}-y^{*}, x-x^{*}\right\rangle \geq 0, & \forall x \in \operatorname{Fix}\left(T_{1}\right), \\ \left\langle\eta F\left(x^{*}\right)+y^{*}-x^{*}, y-y^{*}\right\rangle \geq 0, & \forall y \in \operatorname{Fix}\left(T_{2}\right) .\end{cases}
$$

This problem, which is called bi-level hierarchical optimization problem, was studied by Maingé [20] and Kraikaew et al. [21]. 
(II) In (1.6), if $T_{i}=P_{K_{i}}$ for each $i=1,2$, where $P_{K_{i}}$ is the metric projection from $H$ onto a nonempty closed convex subset $K_{i}$, then it is clear that the $\Omega_{i}=\operatorname{Fix}\left(T_{i}\right)=K_{i}$ and bi-level hierarchical optimization problem (1.6) is equivalent to finding $\left(x^{*}, y^{*}\right) \in K_{1} \times K_{2}$ such that

$$
\begin{cases}\left\langle\rho F\left(y^{*}\right)+x^{*}-y^{*}, x-x^{*}\right\rangle \geq 0, & \forall x \in K_{1}, \\ \left\langle\eta F\left(x^{*}\right)+y^{*}-x^{*}, y-y^{*}\right\rangle \geq 0, & \forall y \in K_{2} .\end{cases}
$$

This system forms a more general problem originated from Nash equilibrium points and it was treated from a theoretical viewpoint in [22-24].

(III) If $\eta=0, \rho>0$ and both sets $\Omega_{1}$ and $\Omega_{2}$ are nonempty closed and convex subsets of $H$, then bi-level hierarchical variational inclusion problem (1.5) reduces to the following (one-level) hierarchical variational inclusion problem:

Find $x^{*} \in \Omega_{1}$ such that for a given positive real number $\rho$, the following inequality holds:

$$
\left\langle\rho F\left(y^{*}\right), x-x^{*}\right\rangle \geq 0, \quad \forall x \in \Omega_{1} .
$$

(IV) If $K_{1}=K_{2}=K$ and $\eta=0, \rho>0$, then (1.7) reduces to the classic variational inequality, i.e., the problem of finding $x^{*} \in K$ such that

$$
\left\langle F\left(x^{*}\right), x-x^{*}\right\rangle \geq 0, \quad \forall x \in K
$$

In (1.5), it is worth noting that if $\Omega_{1}, \Omega_{2}$ are nonempty closed convex subsets in $H$, then the metric projections $P_{\Omega_{1}}$ and $P_{\Omega_{2}}$ from $H$ onto $\Omega_{1}$ and $\Omega_{1}$, respectively, are well defined and problem (1.5) is equivalent to the problem of finding $\left(x^{*}, y^{*}\right) \in \Omega_{1} \times \Omega_{2}$ such that

$$
\left\{\begin{array}{l}
x^{*}=P_{\Omega_{1}}\left[y^{*}-\rho F\left(y^{*}\right)\right], \\
y^{*}=P_{\Omega_{2}}\left[x^{*}-\eta F\left(x^{*}\right)\right] .
\end{array}\right.
$$

However, in practice, both solution sets $\Omega_{1}$ and $\Omega_{2}$ (and hence the two projections) are not given explicitly.

To overcome this drawback, inspired by the method studied by Yamada et al. [25, 26], Maingé [20] and Kraikaew et al. [21], we investigate a more general variant of the scheme proposed by Maingé [20], Kraikaew et al. [21] to replace the projection by some suitable mappings with a nice fixed point set. This strategy also suggests an effective approximation process. Our analysis and method allow us to prove the existence and approximation of solutions to problem (1.5). As applications, we utilize the main results to study the quadratic minimization problems and convex programming problems in Hilbert spaces. The results presented in the paper extend and improve the corresponding results in $[20,21,25,26]$ and others.

\section{Preliminaries}

For the sake of convenience, we first recall some definitions and lemmas for our main results. 
Definition 2.1 A mapping $A: H \rightarrow H$ is said to be $\alpha$-inverse-strongly monotone if there exists $\alpha>0$ such that

$$
\langle A x-A y, x-y\rangle \geq \alpha\|A x-A y\|^{2}, \quad \forall x, y \in H .
$$

A multi-valued mapping $M: H \rightarrow 2^{H}$ is called monotone if for all $x, y \in H, u \in M x$ and $v \in M y$ imply that

$$
\langle u-v, x-y\rangle \geq 0 .
$$

A multi-valued mapping $M: H \rightarrow 2^{H}$ is said to be maximal monotone if it is monotone and for any $(x, u) \in H \times H$,

$$
\langle u-v, x-y\rangle \geq 0
$$

for every $(y, v) \in \operatorname{Graph}(M)$ (the graph of mapping $M$ ) implies that $u \in M x$.

Lemma 2.2 [19] Let $A: H \rightarrow H$ be an $\alpha$-inverse-strongly monotone mapping. Then

(i) $A$ is an $\frac{1}{\alpha}$-Lipschitz continuous and monotone mapping;

(ii) For any constant $\lambda>0$, we have

$$
\|(I-\lambda A) x-(I-\lambda A) y\|^{2} \leq\|x-y\|^{2}+\lambda(\lambda-2 \alpha)\|A x-A y\|^{2} ;
$$

(iii) If $\lambda \in(0,2 \alpha]$, then $I-\lambda A$ is a nonexpansive mapping, where $I$ is the identity mapping on $H$.

Let $H$ be a real Hilbert space, $C$ be a nonempty closed convex subset of $H$. For each $x \in H$, there exists a unique nearest point in $C$, denoted by $P_{C}(x)$, such that

$$
\left\|x-P_{C} x\right\| \leq\|x-y\|, \quad \forall y \in C .
$$

Such a mapping $P_{C}$ from $H$ onto $C$ is called the metric projection.

Remark 2.3 It is well known that the metric projection $P_{C}$ has the following properties:

(i) $P_{C}: H \rightarrow C$ is nonexpansive;

(ii) $P_{C}$ is firmly nonexpansive, i.e.,

$$
\left\|P_{C} x-P_{C} y\right\|^{2} \leq\left\langle P_{C} x-P_{C} y, x-y\right\rangle, \quad \forall x, y \in H
$$

(iii) For each $x \in H$,

$$
z=P_{C}(x) \quad \Leftrightarrow \quad\langle x-z, z-y\rangle \geq 0, \quad \forall y \in C .
$$

Definition 2.4 Let $M: H \rightarrow 2^{H}$ be a multi-valued maximal monotone mapping. Then the mapping $J_{M, \lambda}: H \rightarrow H$ defined by

$$
J_{M, \lambda}(u)=(I+\lambda M)^{-1}(u), \quad u \in H
$$


is called the resolvent operator associated with $M$, where $\lambda$ is any positive number and $I$ is the identity mapping.

Proposition 2.5 [19] Let $M: H \rightarrow 2^{H}$ be a multi-valued maximal monotone mapping, and let $A: H \rightarrow H$ be an $\alpha$-inverse-strongly monotone mapping. Then the following conclusions hold.

(i) The resolvent operator $J_{M, \lambda}$ associated with $M$ is single-valued and nonexpansive for all $\lambda>0$.

(ii) The resolvent operator $J_{M, \lambda}$ is 1-inverse-strongly monotone, i.e.,

$$
\left\|J_{M, \lambda}(x)-J_{M, \lambda}(y)\right\|^{2} \leq\left\langle x-y, J_{M, \lambda}(x)-J_{M, \lambda}(y)\right\rangle, \quad \forall x, y \in H
$$

(iii) $u \in H$ is a solution of the variational inclusion (1.1) if and only if $u=J_{M, \lambda}(u-\lambda A u)$, $\forall \lambda>0$, i.e., $u$ is a fixed point of the mapping $J_{M, \lambda}(I-\lambda A)$. Therefore we have

$$
\Omega=\operatorname{Fix}\left(J_{M, \lambda}(I-\lambda A)\right), \quad \forall \lambda>0,
$$

where $\Omega$ is the set of solutions of variational inclusion problem (1.1).

(iv) If $\lambda \in(0,2 \alpha]$, then $\Omega$ is a closed convex subset in $H$.

In the sequel, we denote the strong and weak convergence of a sequence $\left\{x_{n}\right\}$ in $H$ to an element $x \in H$ by $x_{n} \rightarrow x$ and $x_{n} \rightarrow x$, respectively.

Lemma 2.6 [27] For $x, y \in H$ and $\omega \in(0,1)$, the following statements hold:

(i) $\|x+y\|^{2} \leq\|x\|^{2}+2\langle y, x+y\rangle$;

(ii) $\|(1-\omega) x+\omega y\|^{2}=(1-\omega)\|x\|^{2}+\omega\|y\|^{2}-\omega(1-\omega)\|x-y\|^{2}$.

Lemma 2.7 [28] Let $\left\{a_{n}\right\}$ be a sequence of real numbers, and there exists a subsequence $\left\{a_{m_{j}}\right\}$ of $\left\{a_{n}\right\}$ such that $a_{m_{j}}<a_{m_{j}+1}$ for all $j \in N$, where $N$ is the set of all positive integers. Then there exists a nondecreasing sequence $\left\{n_{k}\right\}$ of $N$ such that $\lim _{k \rightarrow \infty} n_{k}=\infty$ and the following properties are satisfied by all (sufficiently large) number $k \in N$ :

$$
a_{n_{k}} \leq a_{n_{k}+1} \text { and } a_{k} \leq a_{n_{k}+1}
$$

In fact, $n_{k}$ is the largest number $n$ in the set $\{1,2, \ldots, k\}$ such that $a_{n}<a_{n+1}$ holds.

Lemma $2.8[21]$ Let $\left\{a_{n}\right\} \subset[0, \infty),\left\{\alpha_{n}\right\} \subset[0,1),\left\{b_{n}\right\} \subset(-\infty,+\infty), \hat{\alpha} \in[0,1)$ be such that

(i) $\left\{a_{n}\right\}$ is a bounded sequence;

(ii) $a_{n+1} \leq\left(1-\alpha_{n}\right)^{2} a_{n}+2 \alpha_{n} \hat{\alpha} \sqrt{a_{n}} \sqrt{a_{n+1}}+\alpha_{n} b_{n}, \forall n \geq 1$;

(iii) whenever $\left\{a_{n_{k}}\right\}$ is a subsequence of $\left\{a_{n}\right\}$ satisfying

$$
\liminf _{k \rightarrow \infty}\left(a_{n_{k}+1}-a_{n_{k}}\right) \geq 0,
$$

it follows that lim $\sup _{k \rightarrow \infty} b_{n_{k}} \leq 0$;

(iv) $\lim _{n \rightarrow \infty} \alpha_{n}=0$ and $\sum_{n=1}^{\infty} \alpha_{n}=\infty$.

Then $\lim _{n \rightarrow \infty} a_{n}=0$. 


\section{Definition 2.9}

(i) A mapping $T: H \rightarrow H$ is said to be nonexpansive if

$$
\|T x-T y\| \leq\|x-y\|, \quad \forall x, y \in H .
$$

(ii) A mapping $T: H \rightarrow H$ is said to be quasi-nonexpansive if $\operatorname{Fix}(T) \neq \emptyset$ and

$$
\|T x-p\| \leq\|x-p\|, \quad \forall x \in H, p \in \operatorname{Fix}(T) .
$$

It should be noted that $T$ is quasi-nonexpansive if and only if $\forall x \in H, p \in \operatorname{Fix}(T)$

$$
\langle x-T x, x-p\rangle \geq \frac{1}{2}\|x-T x\|^{2} .
$$

(iii) A mapping $T: H \rightarrow H$ is said to be strongly quasi-nonexpansive if $T$ is quasi-nonexpansive and

$$
x_{n}-T x_{n} \rightarrow 0
$$

whenever $\left\{x_{n}\right\}$ is a bounded sequence in $H$ and $\left\|x_{n}-p\right\|-\left\|T x_{n}-p\right\| \rightarrow 0$ for some $p \in \operatorname{Fix}(T)$.

Lemma 2.10 Let $M: H \rightarrow 2^{H}$ be a multi-valued maximal monotone mapping, $A: H \rightarrow H$ be an $\alpha$-inverse-strongly monotone mapping and let $\Omega$ be the set of solutions of variational inclusion problem (1.1) and $\Omega \neq \emptyset$. Then the following statements hold.

(i) If $\lambda \in(0,2 \alpha]$, then the mapping $K: H \rightarrow H$ defined by

$$
K:=J_{M, \lambda}(I-\lambda A)
$$

is quasi-nonexpansive, where $I$ is the identity mapping and $J_{M, \lambda}$ is the resolvent operator associated with $M$.

(ii) The mapping $I-K: H \rightarrow H$ is demiclosed at zero, i.e., for any sequence $\left\{x_{n}\right\} \subset H$, if $x_{n} \rightarrow x$ and $(I-K) x_{n} \rightarrow 0$, then $x=K x$.

(iii) For any $\beta \in(0,1)$, the mapping $K_{\beta}$ defined by

$$
K_{\beta}=(1-\beta) I+\beta K
$$

is a strongly quasi-nonexpansive mapping and $\operatorname{Fix}\left(K_{\beta}\right)=\operatorname{Fix}(K)$.

(iv) $I-K_{\beta}, \beta \in(0,1)$ is demiclosed at zero.

Proof (i) Since $\lambda \in(0,2 \alpha]$, it follows from Lemma 2.2(iii) and Proposition 2.5 that the mapping $K$ is nonexpansive and $\Omega=\operatorname{Fix}(K) \neq \emptyset$. This implies that $K$ is quasi-nonexpansive.

(ii) Since $K$ is a nonexpansive mapping on $H, I-K$ is demiclosed at zero.

(iii) It is obvious that $\operatorname{Fix}\left(K_{\beta}\right)=\operatorname{Fix}(K)$ and $K_{\beta}$ is quasi-nonexpansive.

Next we prove that $K_{\beta}, \beta \in(0,1)$ is a strongly quasi-nonexpansive mapping. 
In fact, let $\left\{x_{n}\right\}$ be any bounded sequence in $H$ and let $p \in \operatorname{Fix}\left(K_{\beta}\right)$ be a given point such that

$$
\left\|x_{n}-p\right\|-\left\|K_{\beta} x_{n}-p\right\| \rightarrow 0 .
$$

Now we prove that $\left\|K_{\beta} x_{n}-x_{n}\right\| \rightarrow 0$.

In fact, it follows from (2.4) that

$$
\begin{aligned}
\left\|K_{\beta} x_{n}-p\right\|^{2} & =\left\|x_{n}-p-\beta\left(x_{n}-K x_{n}\right)\right\|^{2} \\
& =\left\|x_{n}-p\right\|^{2}-2 \beta\left\langle x_{n}-p, x_{n}-K x_{n}\right\rangle+\beta^{2}\left\|x_{n}-K x_{n}\right\|^{2} \\
& \leq\left\|x_{n}-p\right\|^{2}-\beta(1-\beta)\left\|x_{n}-K x_{n}\right\|^{2} .
\end{aligned}
$$

Hence from (2.8), we have

$$
\beta(1-\beta)\left\|K x_{n}-x_{n}\right\|^{2} \leq\left\|x_{n}-p\right\|^{2}-\left\|K_{\beta} x_{n}-p\right\|^{2} \rightarrow 0 .
$$

Since $\beta(1-\beta)>0,\left\|K x_{n}-x_{n}\right\| \rightarrow 0$, and so

$$
\left\|K_{\beta} x_{n}-x_{n}\right\|=\beta\left\|K x_{n}-x_{n}\right\| \rightarrow 0 .
$$

(iv) Since $I-K_{\beta}=\beta(I-K)$ and $I-K$ is demi-closed at zero, hence $I-K_{\beta}$ is demi-closed at zero. This completes the proof.

\section{Main results}

Throughout this section we always assume that the following conditions are satisfied:

(C1) $M_{i}: H \rightarrow 2^{H}, i=1,2$, is a multi-valued maximal monotone mapping, $A_{i}: H \rightarrow H$ is an $\alpha$-inverse-strongly monotone mapping and $\Omega_{i}$ is the set of solutions to variational inclusion problem (1.1) with $A=A_{i}, M=M_{i}$ and $\Omega_{i} \neq \emptyset$;

(C2) $K_{i}$ and $K_{i \beta}, \beta \in(0,1), i=1,2$, are the mappings defined by

$$
\left\{\begin{array}{l}
K_{i}:=J_{M, \lambda}\left(I-\lambda A_{i}\right), \quad \lambda \in(0,2 \alpha], \\
K_{i, \beta}=(1-\beta) I+\beta K_{i}, \quad \beta \in(0,1),
\end{array}\right.
$$

respectively.

We have the following result.

Theorem 3.1 Let $A_{i}, M_{i}, \Omega_{i}, K_{i}, K_{i \beta}, i=1,2$, satisfy the conditions $(\mathrm{C} 1)$ and $(\mathrm{C} 2)$, and let $f, g: H \rightarrow H$ be contractions with a contractive constant $h \in(0,1)$. Let $\left\{x_{n}\right\}$ and $\left\{y_{n}\right\}$ be two sequences defined by

$$
\left\{\begin{array}{l}
x_{0}, y_{0} \in H, \\
x_{n+1}=\left(1-\alpha_{n}\right) K_{1, \beta} x_{n}+\alpha_{n} f\left(K_{2, \beta} y_{n}\right), \\
y_{n+1}=\left(1-\alpha_{n}\right) K_{2, \beta} y_{n}+\alpha_{n} g\left(K_{1, \beta} x_{n}\right), \quad n=0,1,2, \ldots,
\end{array}\right.
$$


where $\left\{\alpha_{n}\right\}$ is a sequence in $(0,1)$ satisfying $\alpha_{n} \rightarrow 0$ and $\sum_{n=0}^{\infty} \alpha_{n}=\infty$. Then the sequences $\left\{x_{n}\right\}$ and $\left\{y_{n}\right\}$ converge to $x^{*}$ and $y^{*}$, respectively, where $\left(x^{*}, y^{*}\right) \in \Omega_{1} \times \Omega_{2}$ is the unique solution of the following (bi-level) hierarchical optimization problem:

$$
\begin{cases}\left\langle x^{*}-f\left(y^{*}\right), x-x^{*}\right\rangle \geq 0, & \forall x \in \Omega_{1}, \\ \left\langle y^{*}-g\left(x^{*}\right), y-y^{*}\right\rangle \geq 0, & \forall y \in \Omega_{2} .\end{cases}
$$

Proof (I) First we prove that (3.3) has a unique solution $\left(x^{*}, y^{*}\right) \in \Omega_{1} \times \Omega_{2}$.

Indeed, it follows from Proposition 2.5 and Lemma 2.10 that both sets $\Omega_{1}, \Omega_{2}$ are nonempty closed and convex and $\Omega_{i}=\operatorname{Fix}\left(K_{i}\right)$ for each $i=1,2$. Hence the metric projection $P_{\Omega_{i}}$ for each $i=1,2$ is well defined. It is clear that the mapping

$$
P_{\Omega_{1}} \circ f \circ P_{\Omega_{2}} \circ g: H \rightarrow H
$$

is a contraction. By the Banach contractive mapping principle, there exists a unique element $x^{*} \in H$ such that

$$
x^{*}=\left(P_{\Omega_{1}} \circ f \circ P_{\Omega_{2}} \circ g\right)\left(x^{*}\right) .
$$

Letting $y^{*}=P_{\Omega_{2}} \circ g\left(x^{*}\right)$, then it is easy to see that

$$
x^{*}=\left(P_{\Omega_{1}} \circ f\right)\left(y^{*}\right), \quad y^{*}=\left(P_{\Omega_{2}} \circ g\right)\left(x^{*}\right)
$$

are the unique solution of (3.3).

(II) Now we prove that $\left\{x_{n}\right\}$ and $\left\{y_{n}\right\}$ are bounded.

In fact, it follows from Lemma 2.10 that $K_{i, \beta}, i=1,2$, is strongly quasi-nonexpansive and $\operatorname{Fix}\left(K_{i, \beta}\right)=\operatorname{Fix}\left(K_{i}\right)=\Omega_{i}$. Since $f$ is $h$-contractive and $x^{*} \in \operatorname{Fix}\left(K_{1, \beta}\right), y^{*} \in \operatorname{Fix}\left(K_{2, \beta}\right)$, we have

$$
\begin{aligned}
\left\|x_{n+1}-x^{*}\right\| & \leq\left(1-\alpha_{n}\right)\left\|K_{1, \beta} x_{n}-x^{*}\right\|+\alpha_{n}\left\|f\left(K_{2, \beta} y_{n}\right)-x^{*}\right\| \\
& \leq\left(1-\alpha_{n}\right)\left\|x_{n}-x^{*}\right\|+\alpha_{n}\left\|f\left(K_{2, \beta} y_{n}\right)-f\left(y^{*}\right)\right\|+\alpha_{n}\left\|f\left(y^{*}\right)-x^{*}\right\| \\
& \leq\left(1-\alpha_{n}\right)\left\|x_{n}-x\right\|+\alpha_{n} h\left\|K_{2, \beta} y_{n}-y^{*}\right\|+\alpha_{n}\left\|f\left(y^{*}\right)-x^{*}\right\| \\
& \leq\left(1-\alpha_{n}\right)\left\|x_{n}-x^{*}\right\|+\alpha_{n} h\left\|y_{n}-y^{*}\right\|+\alpha_{n}\left\|f\left(y^{*}\right)-x^{*}\right\| .
\end{aligned}
$$

Similarly, we can also prove that

$$
\left\|y_{n+1}-y^{*}\right\| \leq\left(1-\alpha_{n}\right)\left\|y_{n}-y^{*}\right\|+\alpha_{n} h\left\|x_{n}-x^{*}\right\|+\alpha_{n}\left\|g\left(x^{*}\right)-y^{*}\right\| .
$$

This implies that

$$
\begin{aligned}
& \left\|x_{n+1}-x^{*}\right\|+\left\|y_{n+1}-y^{*}\right\| \\
& \leq \quad\left(1-\alpha_{n}(1-h)\right)\left(\left\|x_{n}-x^{*}\right\|+\left\|y_{n}-y^{*}\right\|\right) \\
& \quad+\alpha_{n}(1-h) \frac{\left\|f\left(y^{*}\right)-x^{*}\right\|+\left\|g\left(x^{*}\right)-y^{*}\right\|}{1-h} \\
& \quad \leq \max \left\{\left(\left\|x_{n}-x^{*}\right\|+\left\|y_{n}-y^{*}\right\|\right), \frac{\left\|f\left(y^{*}\right)-x^{*}\right\|+\left\|g\left(x^{*}\right)-y^{*}\right\|}{1-h}\right\} .
\end{aligned}
$$


By induction, we have

$$
\begin{aligned}
& \left\|x_{n}-x^{*}\right\|+\left\|y_{n}-y^{*}\right\| \\
& \quad \leq \max \left\{\left(\left\|x_{0}-x^{*}\right\|+\left\|y_{0}-y^{*}\right\|\right), \frac{\left\|f\left(y^{*}\right)-x^{*}\right\|+\left\|g\left(x^{*}\right)-y^{*}\right\|}{1-h}\right\}, \quad \forall n \geq 1 .
\end{aligned}
$$

This implies that $\left\{x_{n}\right\}$ and $\left\{y_{n}\right\}$ are bounded. Consequently, the sequences $\left\{K_{1, \beta} x_{n}\right\}$ and $\left\{K_{2, \beta} y_{n}\right\}$ both are bounded.

(III) Next we prove that for each $n \geq 1$ the following inequality holds.

$$
\begin{aligned}
& \left\|x_{n+1}-x^{*}\right\|^{2}+\left\|y_{n+1}-y^{*}\right\|^{2} \\
& \leq \quad\left(1-\alpha_{n}\right)^{2}\left(\left\|x_{n}-x^{*}\right\|^{2}+\left\|y_{n}-y^{*}\right\|^{2}\right) \\
& \quad+2 \alpha_{n} h\left(\left\|x_{n+1}-x^{*}\right\|\left\|y_{n}-y^{*}\right\|+\left\|x_{n}-x^{*}\right\|\left\|y_{n+1}-y^{*}\right\|\right) \\
& \quad+2 \alpha_{n}\left(\left\langle f\left(y^{*}\right)-x^{*}, x_{n+1}-x^{*}\right\rangle+\left\langle g\left(x^{*}\right)-y^{*}, x_{n+1}-y^{*}\right\rangle\right) .
\end{aligned}
$$

In fact, it follows from (3.2) and Lemma 2.6(i) that

$$
\begin{aligned}
\left\|x_{n+1}-x^{*}\right\|^{2}= & \left\|\left(1-\alpha_{n}\right)\left(K_{1, \beta} x_{n}-x^{*}\right)+\alpha_{n}\left(f\left(K_{2, \beta} y_{n}\right)-x^{*}\right)\right\|^{2} \\
\leq & \left\|\left(1-\alpha_{n}\right)\left(K_{1, \beta} x_{n}-x^{*}\right)\right\|^{2}+2 \alpha_{n}\left(\left(f\left(K_{2, \beta} y_{n}\right)-x^{*}\right), x_{n+1}-x^{*}\right\rangle \\
= & \left(1-\alpha_{n}\right)^{2}\left\|K_{1, \beta} x_{n}-x^{*}\right\|^{2}+2 \alpha_{n}\left\langle f\left(K_{2, \beta} y_{n}\right)-f\left(y^{*}\right), x_{n+1}-x^{*}\right\rangle \\
& +2 \alpha_{n}\left\langle f\left(y^{*}\right)-x^{*}, x_{n+1}-x^{*}\right\rangle \\
\leq & \left(1-\alpha_{n}\right)^{2}\left\|x_{n}-x^{*}\right\|^{2}+2 \alpha_{n}\left\|f\left(K_{2, \beta} y_{n}\right)-f\left(y^{*}\right)\right\|\left\|x_{n+1}-x^{*}\right\| \\
& +2 \alpha_{n}\left\langle f\left(y^{*}\right)-x^{*}, x_{n+1}-x^{*}\right\rangle \\
\leq & \left(1-\alpha_{n}\right)^{2}\left\|x_{n}-x^{*}\right\|^{2}+2 \alpha_{n} h\left\|y_{n}-y^{*}\right\|\left\|x_{n+1}-x^{*}\right\| \\
& +2 \alpha_{n}\left\langle f\left(y^{*}\right)-x^{*}, x_{n+1}-x^{*}\right\rangle .
\end{aligned}
$$

Similarly, we have

$$
\begin{aligned}
\left\|y_{n+1}-y^{*}\right\|^{2} \leq & \left(1-\alpha_{n}\right)^{2}\left\|y_{n}-y^{*}\right\|^{2}+2 \alpha_{n} h\left\|x_{n}-x^{*}\right\|\left\|y_{n+1}-y^{*}\right\| \\
& +2 \alpha_{n}\left|g\left(x^{*}\right)-y^{*}, y_{n+1}-y^{*}\right\rangle .
\end{aligned}
$$

Adding up the last two inequalities, the inequality (3.4) is proved.

(IV) Next we prove the following fact.

If there exists a subsequence $\left\{n_{k}\right\} \subset\{n\}$ such that

$$
\liminf _{k \rightarrow \infty}\left(\left\|x_{n_{k}+1}-x^{*}\right\|^{2}+\left\|y_{n_{k}+1}-y^{*}\right\|^{2}-\left(\left\|x_{n_{k}}-x^{*}\right\|^{2}+\left\|y_{n_{k}}-y^{*}\right\|^{2}\right)\right) \geq 0,
$$

then

$$
\limsup _{k \rightarrow \infty}\left(\left\langle f\left(y^{*}\right)-x^{*}, x_{n_{k}+1}-x^{*}\right\rangle+\left\langle g\left(x^{*}\right)-y^{*}, y_{n_{k}+1}-y^{*}\right\rangle\right) \leq 0 .
$$


In fact, since the norm $\|\cdot\|^{2}$ is convex and $\lim _{n \rightarrow \infty} \alpha_{n}=0$, from (3.2) we have that

$$
\begin{aligned}
0 \leq & \liminf _{k \rightarrow \infty}\left\{\left\|x_{n_{k}+1}-x^{*}\right\|^{2}+\left\|y_{n_{k}+1}-y^{*}\right\|^{2}-\left(\left\|x_{n_{k}}-x^{*}\right\|^{2}+\left\|y_{n_{k}}-y^{*}\right\|^{2}\right)\right\} \\
\leq & \liminf _{k \rightarrow \infty}\left\{\left(1-\alpha_{n_{k}}\right)\left\|K_{1, \beta} x_{n_{k}}-x^{*}\right\|^{2}+\alpha_{n_{k}}\left\|f\left(K_{2, \beta} y_{n_{k}}\right)-x^{*}\right\|^{2}\right. \\
& \left.+\left(1-\alpha_{n_{k}}\right)\left\|K_{2, \beta} y_{n_{k}}-y^{*}\right\|^{2}+\alpha_{n_{k}}\left\|g\left(K_{1, \beta} x_{n_{k}}\right)-y^{*}\right\|^{2}-\left\|x_{n_{k}}-x^{*}\right\|^{2}-\left\|y_{n_{k}}-y^{*}\right\|^{2}\right\} \\
= & \liminf _{k \rightarrow \infty}\left\{\left(\left\|K_{1, \beta} x_{n_{k}}-x^{*}\right\|^{2}-\left\|x_{n_{k}}-x^{*}\right\|^{2}\right)+\left(\left\|K_{2, \beta} y_{n_{k}}-y^{*}\right\|^{2}-\left\|y_{n_{k}}-y^{*}\right\|^{2}\right)\right\} \\
\leq & \limsup _{k \rightarrow \infty}\left\{\left(\left\|K_{1, \beta} x_{n_{k}}-x^{*}\right\|^{2}-\left\|x_{n_{k}}-x^{*}\right\|^{2}\right)+\left(\left\|K_{2, \beta} y_{n_{k}}-y^{*}\right\|^{2}-\left\|y_{n_{k}}-y^{*}\right\|^{2}\right)\right\} \\
= & \limsup _{k \rightarrow \infty}\left\{\left(\left\|K_{1, \beta} x_{n_{k}}-x^{*}\right\|+\left\|x_{n_{k}}-x^{*}\right\|\right)\left(\left\|K_{1, \beta} x_{n_{k}}-x^{*}\right\|-\left\|x_{n_{k}}-x^{*}\right\|\right)\right. \\
& \left.+\left(\left\|K_{2, \beta} y_{n_{k}}-y^{*}\right\|+\left\|y_{n_{k}}-y^{*}\right\|\right)\left(\left\|K_{2, \beta} y_{n_{k}}-y^{*}\right\|-\left\|y_{n_{k}}-y^{*}\right\|\right)\right\} \\
\leq & 0 .
\end{aligned}
$$

The above conclusion can be proved as follows.

Indeed, since the sequences $\left\{\left\|K_{1, \beta} x_{n_{k}}-x^{*}\right\|+\left\|x_{n_{k}}-x^{*}\right\|\right\}$ and $\left\{\left\|K_{2, \beta} y_{n_{k}}-y^{*}\right\|+\left\|y_{n_{k}}-y^{*}\right\|\right\}$ are bounded, and $K_{i, \beta}, i=1,2$, is quasi-nonexpansive, we have

$$
\begin{aligned}
& \left\|K_{1, \beta} x_{n_{k}}-x^{*}\right\| \leq\left\|x_{n_{k}}-x^{*}\right\|, \\
& \left\|K_{2, \beta} y_{n_{k}}-y^{*}\right\| \leq\left\|y_{n_{k}}-y^{*}\right\| .
\end{aligned}
$$

The conclusion is proved. Therefore we have that

$$
\lim _{k \rightarrow \infty}\left(\left\|K_{1, \beta} x_{n_{k}}-x^{*}\right\|-\left\|x_{n_{k}}-x^{*}\right\|\right)=\lim _{k \rightarrow \infty}\left(\left\|K_{2, \beta} y_{n_{k}}-y^{*}\right\|-\left\|y_{n_{k}}-y^{*}\right\|\right)=0
$$

By Lemma 2.10(iii), the mapping $K_{i, \beta}, i=1,2$, is strongly quasi-nonexpansive. Hence from (3.5) we have that

$$
K_{1, \beta} x_{n_{k}}-x_{n_{k}} \rightarrow 0, \quad K_{2, \beta} y_{n_{k}}-y_{n_{k}} \rightarrow 0
$$

This together with (3.2) shows that

$$
x_{n_{k}+1}-x_{n_{k}} \rightarrow 0 \text { and } y_{n_{k}+1}-y_{n_{k}} \rightarrow 0
$$

Since $\left\{x_{n_{k}}\right\}$ is bounded and $H$ is reflexive, there exists a subsequence $\left\{x_{n_{k_{l}}}\right\} \subset\left\{x_{n_{k}}\right\}$ such that $x_{n_{k_{l}}} \rightarrow u$ and

$$
\lim _{l \rightarrow \infty}\left\langle f\left(y^{*}\right)-x^{*}, x_{n_{k_{l}}}-x^{*}\right\rangle=\limsup _{k \rightarrow \infty}\left\langle f\left(y^{*}\right)-x^{*}, x_{n_{k}}-x^{*}\right\rangle=\limsup _{k \rightarrow \infty}\left\langle f\left(y^{*}\right)-x^{*}, x_{n_{k}+1}-x^{*}\right\rangle .
$$

On the other hand, by virtue of Lemma 2.10(iv), $I-K_{1, \beta}$ is demiclosed at zero, and so $u \in \operatorname{Fix}\left(K_{1, \beta}\right)=\Omega_{1}$. Hence from (3.3) we have

$$
\lim _{l \rightarrow \infty}\left\langle f\left(y^{*}\right)-x^{*}, x_{n_{k_{l}}}-x^{*}\right\rangle=\left\langle f\left(y^{*}\right)-x^{*}, u-x^{*}\right\rangle \leq 0
$$


Consequently,

$$
\limsup _{k \rightarrow \infty}\left\{f\left(y^{*}\right)-x^{*}, x_{n_{k}+1}-x^{*}\right\rangle \leq 0
$$

Similarly, by using the same argument, we have

$$
\limsup _{k \rightarrow \infty}\left\langle g\left(x^{*}\right)-y^{*}, y_{n_{k}+1}-y^{*}\right\rangle \leq 0
$$

The desired inequality is proved.

(V) Finally we prove that the sequences $\left\{x_{n}\right\}$ and $\left\{y_{n}\right\}$ defined by (3.2) converge to $x^{*}$ and $y^{*}$, respectively.

It is easy to see that

$$
\begin{aligned}
& \left\|x_{n+1}-x^{*}\right\|\left\|y_{n}-y^{*}\right\|+\left\|x_{n}-x^{*}\right\|\left\|y_{n+1}-y^{*}\right\| \\
& \quad \leq\left(\left\|y_{n}-y^{*}\right\|^{2}+\left\|x_{n}-x^{*}\right\|^{2}\right)^{\frac{1}{2}}\left(\left\|y_{n+1}-y^{*}\right\|^{2}+\left\|x_{n+1}-x^{*}\right\|^{2}\right)^{\frac{1}{2}} .
\end{aligned}
$$

Substituting (3.7) into (3.4), simplifying and putting

$$
\begin{aligned}
& a_{n}:=\left\|x_{n}-x^{*}\right\|^{2}+\left\|y_{n}-y^{*}\right\|^{2}, \\
& b_{n}:=2\left(\left\langle f\left(y^{*}\right)-x^{*}, x_{n+1}-x^{*}\right\rangle+\left\langle g\left(x^{*}\right)-y^{*}, x_{n+1}-y^{*}\right\rangle\right),
\end{aligned}
$$

then we have the following conclusions:

(i) By (II), $\left\{a_{n}\right\}$ is a bounded sequence;

(ii) From (3.4), $a_{n+1} \leq\left(1-\alpha_{n}\right)^{2} a_{n}+2 \alpha_{n} h \sqrt{a_{n}} \sqrt{a_{n+1}}+\alpha_{n} b_{n}, \forall n \geq 1$;

(iii) By (IV), for any subsequence $\left\{a_{n_{k}}\right\} \subset\left\{a_{n}\right\}$ satisfying

$$
\liminf _{k \rightarrow \infty}\left(a_{n_{k}+1}-a_{n_{k}}\right) \geq 0,
$$

it follows that $\lim \sup _{k \rightarrow \infty} b_{n_{k}} \leq 0$.

Hence it follows from Lemma 2.8 that $x_{n} \rightarrow x^{*}$ and $y_{n} \rightarrow y^{*}$. This completes the proof of Theorem 3.1.

Definition 3.2 A mapping $F: H \rightarrow H$ is said to be $\mu$-Lipschitzian and $r$-strongly monotone, if there exist constants $\mu>0$ and $r>0$ such that

$$
\|F x-F y\| \leq \mu\|x-y\|, \quad\langle F x-F y, x-y\rangle \geq r\|x-y\|^{2}, \quad \forall x, y \in H .
$$

Remark 3.3 It is easy to prove that if $F: H \rightarrow H$ is a $\mu$-Lipschitzian and $r$-strongly monotone mapping and if $\rho \in\left(0, \frac{2 r}{\mu^{2}}\right)$, then the mapping $f:=I-\rho F: H \rightarrow H$ is a contraction.

Now we are in a position to prove the following main result.

Theorem 3.4 Let $A_{i}, M_{i}, \Omega_{i}, K_{i}, K_{i \beta}, i=1,2$, be the same as in Theorem 3.1. Let $F: H \rightarrow H$ be a $\mu$-Lipschitzian and r-strongly monotone mapping. Let $\left\{x_{n}\right\}$ and $\left\{y_{n}\right\}$ be the sequences 
defined by

$$
\left\{\begin{array}{l}
x_{0}, y_{0} \in H, \\
x_{n+1}=\left(1-\alpha_{n}\right) K_{1, \beta} x_{n}+\alpha_{n} f\left(K_{2, \beta} y_{n}\right), \\
y_{n+1}=\left(1-\alpha_{n}\right) K_{2, \beta} y_{n}+\alpha_{n} g\left(K_{1, \beta} x_{n}\right),
\end{array}\right.
$$

where $f:=I-\rho F, g:=I-\eta F$ with $\rho, \eta \in\left(0, \frac{2 r}{\mu^{2}}\right), \beta \in(0,1)$ and $\left\{\alpha_{n}\right\}$ is a sequence in $(0,1)$ satisfying the following conditions:

$$
\lim _{n \rightarrow \infty} \alpha_{n}=0, \quad \sum_{n=0}^{\infty} \alpha_{n}=\infty
$$

Then the sequence $\left(\left\{x_{n}\right\},\left\{y_{n}\right\}\right)$ converges strongly to the unique solution $\left(x^{*}, y^{*}\right)$ of bi-level hierarchical variational inclusion problem (1.5).

Proof Indeed, it follows from Remark 3.3 that both mappings $f, g: H \rightarrow H$ are contractive. Therefore all the conditions in Theorem 3.1 are satisfied. By Theorem 3.1, the sequence $\left(\left\{x_{n}\right\},\left\{y_{n}\right\}\right)$ converges strongly to $\left(x^{*}, y^{*}\right) \in \Omega_{1} \times \Omega_{2}$, which is the unique solution of the following bi-level hierarchical optimization problem:

$$
\begin{cases}\left\langle x^{*}-f\left(y^{*}\right), x-x^{*}\right\rangle \geq 0, & \forall x \in \Omega_{1}, \\ \left\langle y^{*}-g\left(x^{*}\right), y-y^{*}\right\rangle \geq 0, & \forall y \in \Omega_{2} .\end{cases}
$$

Since $f=I-\rho F$ and $g=I-\eta F$, we have

$$
\begin{cases}\left\langle\rho F\left(y^{*}\right)+x^{*}-y^{*}, x-x^{*}\right\rangle \geq 0, & \forall x \in \Omega_{1}, \\ \left\langle\eta F\left(x^{*}\right)+y^{*}-x^{*}, y-y^{*}\right\rangle \geq 0, & \forall y \in \Omega_{2} .\end{cases}
$$

This implies that the sequence $\left(\left\{x_{n}\right\},\left\{y_{n}\right\}\right)$ converges strongly to $\left(x^{*}, y^{*}\right) \in \Omega_{1} \times \Omega_{2}$, which is the unique solution of bi-level hierarchical variational inclusion problem (1.5). This completes the proof of Theorem 3.4.

\section{Some applications}

In this section, we shall utilize Theorem 3.1 and Theorem 3.4 to study the convex mathematical programming problem and quadratic minimization problem.

(I) Applications to convex mathematical programming problems.

Let $\psi: H \rightarrow \mathcal{R}$ be a convex and lower semi-continuous function with $\nabla \psi$ being $\mu$ Lipschitzian and $r$-strongly monotone, i.e., it satisfies the following conditions:

$$
\|\nabla \psi(x)-\nabla \psi(y)\| \leq \mu\|x-y\|, \quad \forall x, y \in H, \mu>0,
$$

and

$$
\langle\nabla \psi(x)-\nabla \psi(y), x-y| \geq r\|x-y\|^{2}, \quad \forall x, y \in H, r>0 .
$$


In (1.5) taking $\eta=0, \rho \in\left(0, \frac{2 r}{\mu^{2}}\right)$ and $F=\nabla \psi$, then hierarchical variational inclusion problem (1.5) reduces to the following problem:

Find a point $x^{*} \in \Omega_{1}$ such that

$$
\left\langle\nabla \psi\left(x^{*}\right), x-x^{*}\right\rangle \geq 0, \quad \forall x \in \Omega_{1}
$$

By using the subdifferential inequality, this implies that

$$
\psi(x)-\psi\left(x^{*}\right) \geq\left\langle\nabla \psi\left(x^{*}\right), x-x^{*}\right\rangle \geq 0, \quad \forall x \in \Omega_{1} .
$$

Therefore we have

$$
\psi(x)-\psi\left(x^{*}\right) \geq 0, \quad \forall x \in \Omega_{1} .
$$

Thus problem (4.3) reduces to the convex mathematical programming problem on $\Omega_{1}$ :

Find a point $x^{*} \in \Omega_{1}$ such that

$$
\min _{x \in \Omega_{1}} \psi(x)
$$

Hence, we have the following result.

Theorem 4.1 Let $A_{1}, M_{1}, \Omega_{1}, K_{1}, K_{1, \beta},\left\{\alpha_{n}\right\}$ be the same as in Theorem 3.4. Let $\left\{x_{n}\right\}$ be the iterative sequence defined by

$$
\left\{\begin{array}{l}
x_{0} \in H, \\
x_{n+1}=\left(1-\alpha_{n}\right) K_{1, \beta} x_{n}+\alpha_{n}(I-\rho F)\left(K_{1, \beta} x_{n}\right),
\end{array}\right.
$$

where $\rho \in\left(0, \frac{2 r}{\mu^{2}}\right), \beta \in(0,1)$. Then $\left\{x_{n}\right\}$ converges strongly to $x^{*} \in \Omega_{1}$, which is the unique solution of convex mathematical programming problem (4.5).

(II) Applications to quadratic minimization problems.

Recall that a linear bounded operator $T: H \rightarrow H$ is said to be $\xi$-strongly positive if there exists a positive constant $\xi$ such that

$$
\langle T x, x\rangle \geq \xi\|x\|^{2}, \quad \forall x \in H .
$$

Lemma 4.2 Let $T: H \rightarrow H$ be a $\xi$-strongly positive linear operator and let $\gamma$ be a positive number with $\gamma<\frac{1}{\|T\|}$, where $\|T\|$ is the norm of $T$ defined by

$$
\|T\|=\sup \{\langle T u, u\rangle: u \in H,\|u\|=1\} .
$$

Then we have

(1) The linear operator $F:=I+\gamma T: H \rightarrow H$ is $\mu$-Lipschitzian and $r$-strongly monotone, where $\mu=(1+\gamma\|T\|)$ and $r=1+\gamma \xi$.

(2) If $\rho \in\left(0, \frac{1}{1+\gamma \xi}\right)$, then the linear operator $(I-\rho(I+\gamma T))$ is contractive with a contractive constant $h:=1-\rho(1+\gamma \xi)$. 
Proof (1) In fact, for any $x, y \in H$, we have

$$
\|(I+\gamma T)(x-y)\| \leq(1+\gamma\|T\|)\|x-y\|=\mu\|x-y\| .
$$

Again, since $T: H \rightarrow H$ is a $\xi$-strongly positive linear operator, we have

$$
\langle(I+\gamma T)(x-y), x-y\rangle \geq(1+\gamma \xi)\|x-y\|^{2}=r\|x-y\|^{2} .
$$

Conclusion (1) is proved.

(2) By the definition of the norm of the bounded linear operator $(I-\rho(I+\gamma T))$, we have

$$
\begin{aligned}
\|I-\rho(I+\gamma T)\| & =\sup \{\langle(I-\rho(I+\gamma T)) u, u\rangle: u \in H,\|u\|=1\} \\
& =\sup \{(1-\rho-\rho \gamma)\langle T u, u\rangle: u \in H,\|u\|=1\} \\
& \leq 1-\rho-\rho \gamma \xi, \quad \forall \rho \in\left(0, \frac{1}{1+\gamma \xi}\right) .
\end{aligned}
$$

Therefore, $(I-\rho(I+\gamma T))$ is contractive with a contractive constant $1-\rho(1+\gamma \xi)$. This completes the proof.

From Theorem 3.4 and Lemma 4.2 we have the following result.

Theorem 4.3 Let $A, M, K, K_{\beta}, \Omega$ and $\left\{\alpha_{n}\right\}$ satisfy the same conditions as given in Theorem 3.4. Let the linear mappings $T$ and F satisfy the same conditions as in Lemma 4.2. Then the sequence $\left\{x_{n}\right\}$ defined by

$$
\left\{\begin{array}{l}
x_{0} \in H, \\
x_{n+1}=\left(1-\alpha_{n}\right) K_{\beta} x_{n}+\alpha_{n}(I-\rho F)\left(K_{\beta} x_{n}\right),
\end{array}\right.
$$

where $\rho \in\left(0, \frac{1}{1+\gamma \xi}\right), \beta \in(0,1)$, converges strongly to $x^{*} \in \Omega_{1}$, which is the unique solution of the hierarchical variational inclusion problem:

$$
\left\langle\rho(I+\gamma T) x^{*}, x-x^{*}\right\rangle \geq 0, \quad \forall x \in \Omega,
$$

that is,

$$
\left\langle(I+\gamma T) x^{*}, x-x^{*}\right\rangle \geq 0, \quad \forall x \in \Omega
$$

Letting $g(x):=\frac{\gamma}{2}\langle T x, x\rangle+\frac{1}{2}\|x\|^{2}$, then it is easy to know that $g: H \rightarrow R^{+}$is a continuous and convex functional and $\partial g\left(x^{*}\right)=(I+\gamma T)\left(x^{*}\right)$. By the subdifferential inequality of $g$, we have

$$
g(x)-g\left(x^{*}\right) \geq\left\langle(I+\gamma T)\left(x^{*}\right), x-x^{*}\right\rangle \geq 0, \quad \forall x \in \Omega .
$$

This implies that $x^{*}$ solves the following quadratic minimization problem:

$$
\min _{x \in \Omega}\left\{\frac{\gamma}{2}\langle T x, x\rangle+\frac{1}{2}\|x\|^{2}\right\}
$$

and $x_{n} \rightarrow x^{*}$. This completes the proof. 


\section{Competing interests}

The authors declare that they have no competing interests.

\section{Authors' contributions}

The main idea of this paper was proposed by JKK. JKK and SC prepared the manuscript initially and performed all the steps of the proof in this research. All authors read and approved the final manuscript.

\section{Author details}

${ }^{1}$ College of Statistics and Mathematics, Yunnan University of Finance and Economics, Kunming, Yunnan 650221, China.

${ }^{2}$ Department of Mathematics Education, Kyungnam University, Changwon, Gyeongnam 631-701, Korea. ${ }^{3}$ Department of Applied Mathematics, The Hong Kong Polytechnic University, Hong Kong, P.R. China.

\section{Acknowledgements}

The second author was supported by the Basic Science Research Program through the National Research Foundation of Korea (NRF) funded by the Ministry of Education, Science and Technology (2012R1A1A2042138).

\section{Received: 22 February 2013 Accepted: 18 June 2013 Published: 8 July 2013}

\section{References}

1. Noor, MA, Noor, Kl: Sensitivity analysis of quasi variational inclusions. J. Math. Anal. Appl. 236, 290-299 (1999)

2. Chang, SS: Set-valued variational inclusions in Banach spaces. J. Math. Anal. Appl. 248, 438-454 (2000)

3. Chang, SS: Existence and approximation of solutions of set-valued variational inclusions in Banach spaces. Nonlinear Anal. 47, 583-594 (2001)

4. Demyanov, VF, Stavroulakis, GE, Polyakova, LN, Panagiotopoulos, PD: Quasidifferentiability and Nonsmooth Modeling in Mechanics, Engineering and Economics. Kluwer Academic, Dordrecht (1996)

5. Zhang, SS, Wang, XR, Lee, HW, Chan, CK: Viscosity method for hierarchical fixed point and variational inequalities with applications. Appl. Math. Mech. 32, 241-250 (2011)

6. Yao, YH, Liou, YC: An implicit extra-gradient method for hierarchical variational inequalities. Fixed Point Theory Appl. 2011, Article ID 697248 (2011). doi:10.1155/2011/697248

7. Chang, SS, Cho, YJ, Kim, JK: Hierarchical variational inclusion problems in Hilbert spaces with applications J. Nonlinear Convex Anal. 13, 503-513 (2012)

8. Xu, HK: Viscosity methods for hierarchical fixed point approach to variational inequalities. Taiwan. J. Math. 14, 463-478 (2010)

9. Cianciaruso, F, Colao, V, Muglia, L, Xu, HK: On a implicit hierarchical fixed point approach to variational inequalities. Bull. Aust. Math. Soc. 80, 117-124 (2009)

10. Cianciaruso, F, Marino, G, Muglia, L, Yao, Y: On a two-steps algorithm for hierarchical fixed points and variational inequalities. J. Inequal. Appl. 2009, Article ID 208692 (2009). doi:10.1155/2009/208692

11. Kim, JK, Kim, KS: A new system of generalized nonlinear mixed quasivariational inequalities and iterative algorithms in Hilbert spaces. J. Korean Math. Soc. 44, 823-834 (2007)

12. Kim, JK, Kim, KS: New system of generalized mixed variational inequalities with nonlinear mappings in Hilbert spaces. J. Comput. Anal. Appl. 12,601-612 (2010)

13. Kim, JK, Kim, KS: On new system of generalized nonlinear mixed quasivariational inequalities with two-variable operators. Taiwan. J. Math. 11, 867-881 (2007)

14. Chang, SS, Cho, YJ, Kim, JK: On the two-step projection methods and applications to variational inequalities. Math. Inequal. Appl. 10, 755-760 (2007)

15. Mainge, PE, Moudafi, A: Strong convergence of an iterative method for hierarchical fixed point problems. Pac J. Optim. 3, 529-538 (2007)

16. Guo, G, Wang, S, Cho, YJ: Strong convergence algorithms for hierarchical fixed point problems and variational inequalities. J. Appl. Math. 2011, Article ID 164978 (2011). doi:10.1155/2011/164978

17. Yao, Y, Cho, YJ, Liou, YC: Hierarchical convergence of an implicit double-net algorithm for nonexpansive semigroups and variational inequality problems. Fixed Point Theory Appl. 2011, Article ID 101 (2011)

18. Yao, Y, Cho, YJ, Yang, P: An iterative algorithm for a hierarchical problem. J. Appl. Math. 2012, Article ID 320421 (2012). doi:10.1155/2012/320421

19. Chang, SS, Joseph Lee, HW, Chan, CK: Algorithms of common solutions for quasi variational inclusion and fixed point problems. Appl. Math. Mech. 29, 1-11 (2008)

20. Maingé, PE: New approach to solving a system of variational inequalities and hierarchical problems. J. Optim. Theory Appl. 138, 459-477 (2008)

21. Kraikaew, R, Saejung, S: On Maingé's approach for hierarchical optimization problem. J. Optim. Theory Appl. (2012). doi:10.1007/s10957-011-9982-4

22. Kassay, G, Kolumbán, J, Páles, Z: Factorization of Minty and Stampacchia variational inequality systems. Eur. J. Oper. Res. 143, 377-389 (2002)

23. Kassay, G, Kolumbán, J: System of multi-valued variational inequalities. Publ. Math. (Debr.) 56, 185-195 (2000)

24. Verma, RU: Projection methods, algorithms, and a new system of nonlinear variational inequalities. Comput. Math. Appl. 41, 1025-1031 (2001)

25. Yamada, I, Ogura, N: Hybrid steepest descent method for variational inequality problem over the fixed point set of certain quasi-nonexpansive mappings. Numer. Funct. Anal. Optim. 25, 619-655 (2004)

26. Yamada, I, Ogura, N, Shirakawa, N: A numerically robust hybrid steepest descent method for the convexly constrained generalized inverse problems. In: Nashed, MZ, Scherzer, O (eds.) Inverse Problems, Image Analysis, and Medical Imaging. Contemporary Mathematics, pp. 269-305. Am. Math. Soc., Providence (2002)

27. Takahashi, W: Introduction to Nonlinear and Convex Analysis. Yokohama Publishers, Yokohama (2009)

28. Maingé, PE: The viscosity approximation process for quasi-nonexpansive mappings in Hilbert spaces. Comput. Math. Appl. 59, 74-79 (2010) 
doi:10.1186/1687-1812-2013-179

Cite this article as: Chang et al.: On the hierarchical variational inclusion problems in Hilbert spaces. Fixed Point Theory and Applications 2013 2013:179.

Submit your manuscript to a SpringerOpen ${ }^{\circ}$ journal and benefit from:

- Convenient online submission

- Rigorous peer review

- Immediate publication on acceptance

- Open access: articles freely available online

- High visibility within the field

- Retaining the copyright to your article

Submit your next manuscript at $\gg$ springeropen.com 